\title{
DIFFUSION DES RAYONS X ET DES NEUTRONS
}

\author{
Par R. GRAF et J. M. RAYNAL, \\ Faculté des Sciences de Rouen.
}

\begin{abstract}
Résumé. - Dans un cristal, tout écart de périodicité se traduit par une diffusion anomale des rayons $\mathrm{X}$ et des neutrons. Les auteurs examinent les différents types de désordres et les diffusions correspondantes. Ils décrivent les principales techniques et montrent les avantages présentés par les neutrons.
\end{abstract}

Abstract. - In a crystal, every deviation from the periodicity involves an anomalous scattering of X-rays and of neutrons. The authors examine the various types of disorders and the corresponding scattering. They describe the principal techniques and point out the advantages of using the neutrons.

Dans la théorie élémentaire de la diffraction des rayons $\mathrm{X}$, on considère un cristal parfait au sens strict, c'est-à-dire formé par un ensemble d'atomes immobiles, rangés d'une façon absolument régulière suivant le schéma d'un réseau tridimensionnel.

Un tel cristal donne uniquement des réflexions sélectives ou réflexions de Bragg : dans l'espace réciproque, l'intensité diffractée est localisée aux nœuds du réseau réciproque. Cela suppose toutefois que le cristal comporte un grand nombre de mailles.

Tout écart à la régularité idéale entraîne des réflexions diffuses qui peuvent couvrir des régions plus ou moins étendues de l'espace réciproque. L'étude de ces diffusions fournit des renseignements sur la nature des désordres affectant l'édifice cristallin.

Principales causes de diffusion dans le cas des rayons $\mathbf{X}$. - Les causes des réflexions diffuses sont nombreuses et nous examinerons tout d'abord le cas des rayons $\mathrm{X}[1,2,3]$. Celui des neutrons est d'ailleurs analogue.

A. Agitation thermique. - Elle introduit des irrégularités dynamiques de position des atomes, donnant naissance à une diffusion générale, plus intense au voisinage des nœuds réciproques, mais faible au centre. Dans les applications envisagées en métallurgie, cette diffusion thermique est parasite, mais elle est inévitable même à basse température et doit être déduite des diffusions observées si l'on veut atteindre les désordres statiques.

B. Désordres statiques de substitution. - Ces désordres se manifestent dans les solutions solides de substitution et il faut envisager deux cas :

1. Les solutions solides désordonnées en équilibre thermodynamique. - Si dans un cristal formé d'atomes A

REVUE DE PHYSIQUE APPLIQUÉE. - T. 3. No 4. DÉCEMBRE 1968. on remplace au hasard certains de ces atomes par des atomes $\mathrm{B}$, on obtient une solution solide désordonnée, qui peut être stable dans diverses conditions, par exemple à haute température.

Une telle solution solide donne une diffusion générale dite de Laue, dont l'intensité est proportionnelle à $c(1-c)\left(f_{\mathbf{A}}-f_{\mathbf{B}}\right)^{2}$ où $c$ désigne la concentration en atomes $\mathrm{B}, f_{\mathrm{A}}$ et $f_{\mathrm{B}}$ étant les facteurs de diffusion atomiques, dont la valeur diminue lorsque l'angle de diffusion augmente. Mais le désordre parfait est rare, les atomes B ayant généralement tendance à s'entourer :

- soit d'atomes d'espèce différente $A$, ce qui conduit à un certain ordre à courte distance;

- soit d'atomes de même espèce $B$, ce qui conduit à des petits amas d'atomes $\mathrm{B}$ (clusters).

Dans les deux cas, la diffusion de Laue subit une modulation, avec des maximums situés entre les réflexions de Bragg dans le premier cas, autour de ces réflexions dans le second.

2. Solutions solides hors d'équilibre. - Les solutions solides désordonnées stables à haute température et trempées conservent généralement leur structure désordonnée, mais sont alors en équilibre métastable. Un revenu à température moyenne provoque une évolution soit vers un ordre à grande distance, soit vers la précipitation d'une nouvelle phase :

a) Dans le premier cas, les atomes B viennent se placer dans des sites bien déterminés de la maille. Il en résulte une solution solide ordonnée et il apparaît des réflexions supplémentaires dites de surstructure, dues au fait que la maille du réseau période devient plus grande. Tant que la solution solide n'est que partiellement ordonnée, ces réflexions sont floues et doivent être considérées comme des diffusions. Lorsque la solution solide est parfaitement ordonnée, l'arrange- 
ment atomique est régulier, mais il peut se former des domaines antiphases, dans lesquels la place des atomes $\mathrm{A}$ et $\mathrm{B}$ est intervertie, ce qui entraîne un dédoublement des taches de surstructure.

b) Dans le cas où l'évolution se fait vers la précipitation d'une seconde phase, le premier stade du processus de décomposition est très souvent un rassemblement des atomes B en amas, dont la taille est de l'ordre de $100 \AA$ et qui ont une forme géométrique déterminée : sphère, plaquette, aiguille... Dans ces amas, les atomes $\mathrm{B}$ sont distribués aux nœuds du même réseau cristallin que les atomes $\mathrm{A}$ : ce sont les amas de préprécipitation ou zones de Guinier-Preston. Il en résulte des diffusions, localisées autour des nœuds réciproques de la matrice et près du centre, si toutefois $f_{\mathrm{A}} \neq f_{\mathrm{B}}$. Elles sont la figure réciproque de la forme des amas lorsqu'il n'y a pas d'interaction notable entre ces derniers.

Des diffusions analogues peuvent apparaître dans le cas de très petits précipités dont la structure est distincte de la matrice, mais on les observe également autour des nœuds du précipité.

On peut encore rattacher au cas des zones de Guinier-Preston celui des amas de lacunes, qui donnent le même type de diffusions, surtout visible au centre.

Les dislocations peuvent elles-mêmes être traitées comme des perturbations locales de la densité électronique se répétant dans le cristal et doivent donner une diffusion près du centre. Mais cette diffusion est généralement masquée par le phénomène de double diffraction.

C. DÉsordres statiques de déplaGement. - Dans une solution solide, des distorsions sont généralement dues aux différences de rayon atomique des métaux constituants.

1) Supposons d'abord que $f_{\mathbf{A}}$ et $f_{\mathbf{B}}$ sont très voisins et que la solution solide est désordonnée. La diffusion de Laue est alors nulle, mais il apparaît une diffusion autour des nœuds réciproques de la solution solide, plus intense pour les ordres élevés mais nulle au centre (Huang).

2) Le phénomène est plus complexe lorsque $f_{\mathbf{A}}$ et $f_{\mathbf{B}}$ sont différents : à la diffusion de Laue se superposent alors non seulement les diffusions précédentes, mais également une modulation donnant des maximums de diffusion dont la position par rapport aux nœuds réciproques varie suivant que l'atome le plus gros est également le plus diffusant ou non.

De tels désordres de déplacement peuvent aussi se produire dans les solutions solides contenant des zones de Guinier-Preston : dans ce cas, les distorsions conditionnent la forme des amas (Nabarro) et les diffusions observées sont modifiées : par exemple, les traînées de diffusion données par les zones planes deviennent dissymétriques.

En outre, les désordres combinés de déplacement et de substitution peuvent conduire à une répartition sensiblement périodique des zones de préprécipitation dans la solution solide. Leur interaction est donc importante et les diffusions qui en résultent sont des nœuds satellites, situés près des nœuds réciproques de la matrice. Leur position et leur intensité dépendent de la nature du désordre.

Mais on peut également observer des désordres de déplacement dans les cristaux de métaux purs, par exemple les défauts d'empilement, qui se traduisent par des traînées de diffusion normales au plan des défauts.

Les techniques de diffusion des rayons $\mathbf{X}$. A. Desaription des teghniques. - Ces techniques revêtent des aspects différents suivant la région de l'espace réciproque à explorer. Mais dans tous les cas, on a intérêt à utiliser un rayonnement strictement monochromatique obtenu par réflexion sélective sur un ou deux cristaux, de manière à éliminer le spectre continu. Les longueurs d'onde employées sont généralement celles du cuivre ou du molybdène.

1. Techniques de diffusion centrale. - Il s'agit de la diffusion aux petits angles, c'est-à-dire au voisinage du faisceau direct [4]. La méthode photographique permet d'enregistrer l'allure générale de cette diffusion, sous vide et avec des distances échantillon-film de l'ordre de $100 \mathrm{~mm}$ ( fig. 1). Mais l'interprétation quan-

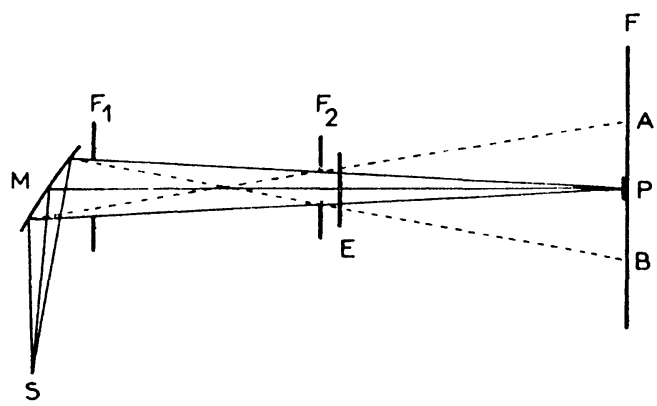

FIG. 1. - Montage de diffusion centrale des rayons $\mathrm{X}$ avec film : $S$, foyer du tube; $F_{1}, F_{2}$, fentes ; $F$, film; $\mathrm{M}$, monochromateur; $\mathrm{E}$, échantillon; $\mathrm{P}$, piège ; $\mathrm{AB}$, zone voilée.

titative des diffusions exige des mesures absolues, qui ne sont possibles qu'avec le diffractomètre à compteur. On peut employer un diffractomètre normal équipé avec des fentes de précision, ou des diffractomètres spéciaux. On explore ainsi le centre de la strate équatoriale. Des corrections s'imposent pour tenir compte de la hauteur de la fente et il faut soustraire la diffusion parasite mesurée dans des conditions comparables sans échantillon.

Parmi les diffractomètres spéciaux, citons le montage intégrant de Levelut [5] avec lequel on enregistre l'intensité diffusée à travers un diaphragme annulaire 


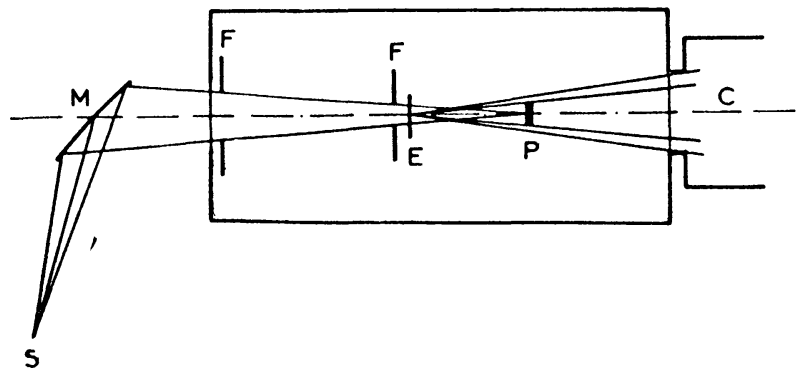

FIG. 2. - Montage intégrant de Levelut : S, foyer ponctuel ; $\mathrm{F}$, fentes ; $\mathrm{P}$, piège ; $\mathrm{M}$, monochromateur à double courbure; E, échantillon; C, compteur à scintillation et photomultiplicateur.

à l'aide d'un compteur à scintillation ( fig. 2). Il permet une grande sensibilité de détection pour les petits amas d'atomes, par différence avec la diffusion de Laue.

2. Techniques pour l'étude des diffusions non centrales. Dans le cas de monocristaux, on peut utiliser une chambre de cristal tournant à film cylindrique de diamètre suffisant, au moins $76 \mathrm{~mm}$ [6]. La répartition des diffusions sur une strate peut être étudiée avec une chambre à film mobile, par exemple un rétigraphe. Mais les mesures quantitatives sur la strate équatoriale ou dans le cas d'échantillons polycristallins se font avantageusement avec le goniomètre à comp-

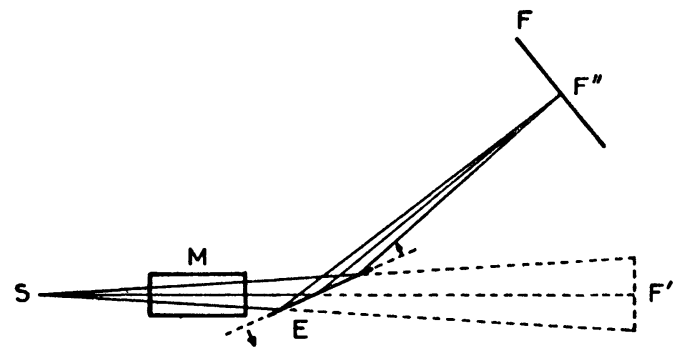

a

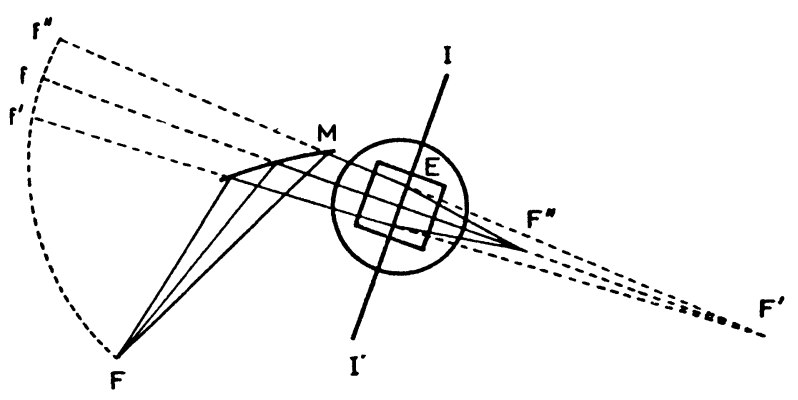

b

FIG. 3. - Montage à double focalisation de Manenc.

a) Vue horizontale : $\mathrm{S}$, foyer ponctuel; E, échantillon ; $\mathrm{F}$, film; $\mathrm{M}$, monochromateur ; $\mathrm{F}^{\prime}$, foyer image $\mathrm{du}$ monochromateur; $\mathrm{F}^{\prime \prime}$, tache focalisée.

b) Vue verticale : $\mathrm{II}^{\prime}$, axe d'oscillation de l'échantillon. teur. Elles doivent généralement être corrigées de la diffusion Compton et de la diffusion thermique.

Notons l'intérêt du montage à double focalisation de Manenc [7] pour l'étude des diffusions au voisinage des taches de Bragg, en particulier celles qui sont dues aux zones de préprécipitation à structure modulée. La première focalisation est assurée par un monochromateur cylindrique et la seconde par l'échantillon -ui-même (fig. 3).

B. Répartition de Ges teghniques en France. A notre connaissance, de telles techniques sont utilisées dans plusieurs laboratoires de métallurgie ou de physique des métaux : Faculté d'Orsay, Faculté de Rouen, Faculté de Poitiers, I.R.S.I.D., O.N.E.R.A. Elles sont généralement appliquées à l'étude des phénomènes de préprécipitation et des phénomènes d'ordre, ainsi qu'à l'étude des défauts créés par irradiation.

La diffusion des neutrons. - La diffraction des neutrons est utilisée depuis quelque temps déjà pour l'étude des structures cristallines, en particulier celles des corps ferromagnétiques [8].

Mais la diffusion des neutrons est une technique très récente. On a surtout envisagé la diffusion centrale des neutrons thermiques lents, qui présente en effet un certain nombre d'avantages sur la diffusion centrale des rayons $\mathrm{X}[9],[10]$ :

1) En raison de la grande longueur d'onde associée à ces neutrons, de 5 à $15 \AA$ environ, on peut éviter la double diffraction de Bragg, car pour les métaux les distances réticulaires ne dépassent généralement pas $3 \AA$. Il est alors possible d'enregistrer la diffusion beaucoup plus près du faisceau direct, donc par exemple d'étudier la diffusion centrale due aux dislocations et d'en déduire la densité de ces dislocations.

2) L'interaction neutron/atome diffusant se produit avec le noyau de cet atome, de sorte que les facteurs de diffusion atomiques des neutrons sont très différents de ceux pour les rayons $\mathrm{X}$ et ne varient pas avec l'angle de diffusion. Il devient alors possible d'étudier la diffusion centrale d'alliages dont les éléments ont des numéros atomiques très voisins comme $\mathrm{Al}-\mathrm{Mg}$, $\mathrm{Fe}-\mathrm{Co} . .$.

3) Malgré leur grande longueur d'onde, les neutrons sont beaucoup moins absorbés que les rayons $\mathrm{X}$ et l'épaisseur optimale des échantillons est relativement considérable : elle est de l'ordre de $20 \mathrm{~mm}$ pour $\mathrm{Al}$ (contre $0,05 \mathrm{~mm}$ pour les rayons $\mathrm{X}$ de $1,5 \AA$ ).

Bien que les sources de neutrons soient comparativement beaucoup moins intenses qu'un tube à rayons $\mathrm{X}$, il en résulte que l'on obtient malgré tout des intensités diffusées notables, d'autant plus que la grande dimension des sources de neutrons permet d'utiliser des fentes hautes et larges, l'augmentation des dimensions du montage permettant de conserver une résolution comparable à celle obtenue avec les rayons $\mathrm{X}$. 


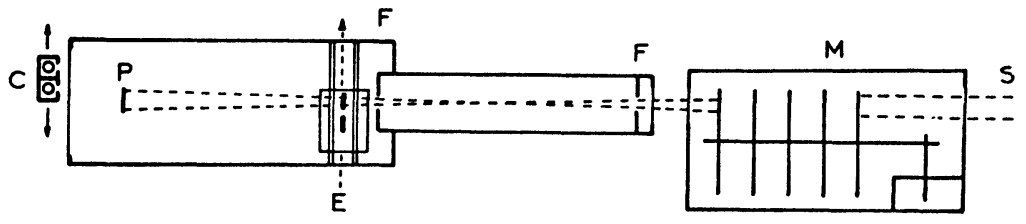

FIG. 4. - Montage de diffusion centrale des neutrons avec compteur :

$\mathrm{S}$, faisceau de neutrons ; F, fentes ; $\mathrm{P}$, pièges ; $\mathrm{M}$, sélecteur de vitesses ; E, échantillons sur chariot mobile ; $\mathrm{C}$, compteur au $\mathrm{BF}_{3}$ sur chariot mobile.

La monochromatisation du faisceau de neutrons est assurée par un sélecteur rotatif donnant une largeur spectrale totale d'environ $1,4 \AA$ pour une longueur d'onde de $7 \AA$. Le faisceau peut être défini par deux fentes ( fig. 4) ou focalisé à l'aide d'un miroir courbé à réflexion totale. La détection se fait avec un compteur au $\mathrm{BF}_{3}$. Plusieurs échantillons sont disposés sur un chariot mobile télécommandé et peuvent être examinés successivement. Une technique d'enregistrement sur film est à l'étude.

Les seuls laboratoires actuellement équipés pour la diffusion centrale des neutrons lents sont situés en Allemagne, à Munich et à Julich. Des essais sont actuellement en cours sur les alliages $\mathrm{Al}-\mathrm{Zn}$ pour comparer les possibilités de cette technique et de la diffusion centrale des rayons $\mathrm{X}$ dans le domaine de la préprécipitation. Les mesures aux neutrons sont effectuées au K.F.A. de Julich, sur la pile FR-J2, les mesures aux rayons $\mathrm{X}$ à Rouen et à Orsay. On envisage d'étudier ultérieurement les alliages ternaires $\mathrm{Al}-\mathrm{Zn}-\mathrm{Mg}$ et d'aborder les binaires Al-Mg.

En résumé, la diffusion des rayons $\mathrm{X}$ et celle des neutrons constituent deux techniques complémentaires; mais alors que la diffusion des rayons $\mathrm{X}$ est une méthode d'étude assez répandue, la diffusion des neutrons est plus rare et mériterait d'être développée car elle permet de résoudre de nombreux problèmes métallurgiques.

\section{BIBLIOGRAPHIE,}

[1] Guinier (A.), L'état solide, Stoops, Bruxelles, 1952, p. 197.

[2] Guinier (A.), Solid State Physics, 1959, 9, 293.

[3] GEROLD (V.), Z. Metallkunde, 1963, 54, 6, 370.

[4] GUINIER (A.) et FouRnE'T (G.), Small angle scattering of X-rays, J. Wiley, New York, 1955.

[5] LeVelut (A. M.), LAMBERT (M.) et Guinier (A.), C. R. Acad. Sc., 1962, 255, 319 .
[6] Wooster (W. A.), Diffuses X-ray reflections from crystals, Clarendon Press, Oxford, 1962.

[7] Manenc (J.), Acta Cryst., 1957, 10, 4, 259.

[8] Ringo (G. R.), Handbuch dev Physik, 32, 552.

[9] SPRinger (T.) et Schmatz (W.), Bull. Soc. franç. Minéral. Cristallogr., 1967, 90, 428.

[10] Christ (J.), Schilling (W.), Schmatz (W.) et SPRINGER (T.), Z. angew. Physik, 1965, 18, 4, 295. 